\title{
Neurofilament Light Chain Levels in Multiple Sclerosis Correlate With Lesions Containing Foamy Macrophages and With Acute Axonal Damage
}

Aletta van den Bosch, MSc, * Nina Fransen, PhD, * Matthew Mason, PhD, Annemieke Johanna Rozemuller, PhD, Charlotte Teunissen, PhD, Joost Smolders, PhD, and Inge Huitinga, PhD

Neurol Neuroimmunol Neuroinflamm 2022;9:e1154. doi:10.1212/NXI.0000000000001154

\section{Abstract}

\section{Background and Objectives}

To investigate whether white matter lesion activity, acute axonal damage, and axonal density in MS associate with CSF neurofilament light chain (NfL) levels.

\section{Methods}

Of 101 brain donors with MS ( $\mathrm{n}=92$ progressive MS, $\mathrm{n}=9$ relapsing-remitting MS), ventricular CSF was collected, and NfL levels were measured. White matter lesions were classified as active, mixed, inactive, or remyelinated, and microglia/macrophage morphology in active and mixed lesions was classified as ramified, ameboid, or foamy. In addition, axonal density and acute axonal damage were assessed using Bielschowsky and amyloid precursor protein (APP) (immune)histochemistry.

\section{Results}

CSF NfL measurements of donors with recent $(<1$ year) or clinically silent stroke were excluded. CSF NfL levels correlated negatively with disease duration $(p=6.9 \mathrm{e}-3, r=0.31)$. In donors without atrophy, CSF NfL levels correlated positively with the proportion of active and mixed lesions containing foamy microglia/macrophages $(p=9.85 \mathrm{e}-10$ and $p=1.75 \mathrm{e}-3$, respectively), but not with those containing ramified microglia. CSF NfL correlated negatively with proportions of inactive $(p=5.66 \mathrm{e}-3)$ and remyelinated lesions $(p=0.03)$. In the normal appearing pyramid tract, axonal density negatively correlated with CSF NfL levels (Bielschowsky, $p=0.02, r=-0.31)$, and the presence of acute axonal damage in lesions was related to higher NfL levels (APP, $p=1.17 e-6$ ). The amount of acute axonal damage was higher in active lesions with foamy microglia/macrophages and in the rim of mixed lesions with foamy microglia/macrophages when compared with active lesions containing ramified microglia/ macrophages ( $p=4.6 \mathrm{e}-3$ and $p=0.02$, respectively), the center and border of mixed lesions containing ramified microglia/macrophages (center: $p=4.6 \mathrm{e}-3$, border, $p=4.6 \mathrm{e}-3$, and n.s., $p=$ $4.6 \mathrm{e}-3$, respectively), the center of mixed lesions containing foamy microglia/macrophages ( $p=$ $4.6 \mathrm{e}-3$ and $p=0.02$, respectively), inactive lesions ( $p=4.6 \mathrm{e}-3$ and $p=4.6 \mathrm{e}-3$, respectively), and remyelinated lesions ( $p=0.03$ and $p=0.04$, respectively).

\section{Discussion}

Our results demonstrated that active and mixed white matter MS lesions with foamy microglia show high acute axonal damage and correlate with elevated CSF NfL levels. Our data support the use of this biomarker to monitor inflammatory demyelinating lesion activity with axonal damage in MS.
Correspondence

Drs. van den Bosch

a.v.d.bosch@nin.knaw.nl

\footnotetext{
*These authors contributed equally to this work and are co-first authors.

From the Neuroimmunology Research Group (A.B., N.F., M.M., J.S., I.H.), Netherlands Institute for Neuroscience; Department Pathology (A.J.R.), Amsterdam UMC; Neurochemistry Lab (C.T.), Department of Clinical Chemistry, Amsterdam Neuroscience, Amsterdam UMC, Vrije Universiteit; Department of Neurology and Immunology (J.S.), MS Center ErasMS, ErasmusMC, Rotterdam; and Swammerdam Institute for Life Sciences (I.H.), University of Amsterdam, the Netherlands.

Go to Neurology.org/NN for full disclosures. Funding information is provided at the end of the article.

The Article Processing Charge was funded by the authors.
}

This is an open access article distributed under the terms of the Creative Commons Attribution-NonCommercial-NoDerivatives License 4.0 (CC BY-NC-ND), which permits downloading and sharing the work provided it is properly cited. The work cannot be changed in any way or used commercially without permission from the journal. 


\section{Glossary}

APP = amyloid precursor protein; EDSS = Expanded Disability Status Scale; GLM = Generalized Linear Model; MMAS = microglia/macrophage activation score; NBB = Netherlands Brain Bank; NfL = neurofilament light chain; SUMI = super mix; TBS $=$ tris buffered saline.

Multiple sclerosis (MS) is a chronic inflammatory disease of the CNS with focal demyelinating lesions throughout the CNS. ${ }^{1,2}$ Pathologically, MS is characterized by different types of lesions that can be staged by the presence and morphology of microglia/macrophages in relation to demyelination. ${ }^{3,4}$ Furthermore, some patients show diffuse atrophy with axonal loss throughout the CNS. ${ }^{3,5} \mathrm{We}$ and others found that at time of death, there is substantial inflammatory lesion activity, ${ }^{6}$ and $57 \%$ of all lesions in the MS autopsy cohort of the Netherlands Brain Bank (NBB) are either active or mixed. ${ }^{3}$ The level of inflammatory lesion activity is correlated with a more severe disease course, ${ }^{3}$ and in this study, we assessed whether axonal damage coincides with the inflammatory lesion activity in the same MS autopsy cohort.

Neurofilaments are neuron-specific structural scaffolding protein components of the cytoskeleton that are essential for axonal growth and maintenance. ${ }^{7}$ During axonal damage, neurofilaments are released into the CSF. Therefore, neurofilaments are a potential molecular fluid biomarker for the extent of axonal damage. ${ }^{8}$ Indeed, levels of neurofilament light chain (NfL) in the CSF or blood plasma/serum have been used as general indicators or predictors of neuronal damage in various neurologic disorders and events, among which are MS, Alzheimer disease, and traumatic brain injury. ${ }^{8-16}$ In MS, NfL can serve as a prognostic biomarker in both relapsing remitting and progressive MS and for monitoring clinical relapses and treatment response. ${ }^{13}$ Previous studies have focused on understanding the value of $\mathrm{NfL}$ measurements in MS by combining conventional MRI measurements and clinical data with NfL in the blood and/ or CSF. ${ }^{17-21} \mathrm{NfL}$ levels correlate with clinical relapses and radiologic biomarkers of inflammatory disease activity because they reflect the amount of gadolinium-enhancing lesions, ${ }^{18,20}$ the amount of paramagnetic rim MRI lesions in the absence of gadolinium-enhancing lesions, ${ }^{22}$ and disease progression regarding the $\mathrm{T} 2$ lesion load, ${ }^{12,20} \mathrm{~T} 2$ lesion volume, ${ }^{18,20}$ and presence of atrophy of the brain and spinal cord. $^{18,21,23}$

These observations raise the question whether neuropathologic hallmarks of inflammatory lesion activity, neuroaxonal damage, or neurodegeneration correlate with axonal damage, as reflected by CSF NfL levels in MS. In this study, we assessed the relationship between CSF NfL levels and disease severity, axonal loss in the normal appearing white matter (NAWM), and white matter lesion characteristics including microglia/macrophage activation score (MMAS) and acute axonal damage, as measured with amyloid precursor protein
(APP) in lesions in a well-characterized MS autopsy cohort of 101 cases of the NBB.

\section{Methods}

\section{Donors}

For inclusion in this study, we screened a total of 182 brain donors with MS that came to autopsy at the NBB between 1991 and $2015 .^{3}$ MS pathology was confirmed by a certified neuropathologist, and donors with clinical or pathologic features of encephalomyelitis were excluded. Donors were also excluded if there were clinical signs of dementia or if a neuropathologist diagnosed pathologic dementia based on senile pathology of $\alpha$-synuclein presence, Tau + tangles, and $\beta$-amyloid plaques (Braak $>2$, Thal fase $>2$ ). Cases of whom no CSF was available for analysis were excluded. The included study cohort consisted of 101 donors with MS ( $\mathrm{n}=92$ progressive MS, $n=9$ relapsing-remitting MS). Clinical disability status was scored following the Kurtze Expanded Disability Status Scale (EDSS), and the time from first symptoms to EDSS- 6 and EDSS- 8 was determined, together with the age at onset and the total duration of disease from onset of first symptoms. Disease severity score was calculated as $5-\log$ (years to EDSS6 + 1). Donors were scored for minor senile pathology (Braak $\leq 2$, Thal fase $\leq 2$ ), a history of stroke in their clinical and postmortem neuropathologic files, and for the presence of atrophy, as based on the postmortem macroscopic examination. The exclusion criteria and studied characteristics that were related to NfL levels of the various subgroups are visualized in the flowchart in Figure 1. Donor demographics are summarized in Table 1.

\section{Donor Consents}

Donors provided informed consent for brain autopsy and for the use of material and clinical data for research purposes in compliance with national ethical guidelines.

\section{Tissue Dissection and Sample Collection}

The NBB autopsy procedures were approved by the Ethical Committee of the VU University Medical Center in Amsterdam, the Netherlands. CSF of all donors was collected from the ventricles and stored at $-80^{\circ} \mathrm{C}$. Of 43 donors, blood was collected from the heart, centrifuged at $3.000 \mathrm{rpm}$ for 15 minutes, and the plasma was aliquoted and stored at $-80^{\circ} \mathrm{C}$. Regarding the higher number of donors with available CSF samples compared with plasma samples, we focused our current analysis on CSF samples. Blocks were dissected as previously described ${ }^{3}$ from standardized locations from the brain stem and the spinal cord and any MS plaques visible 


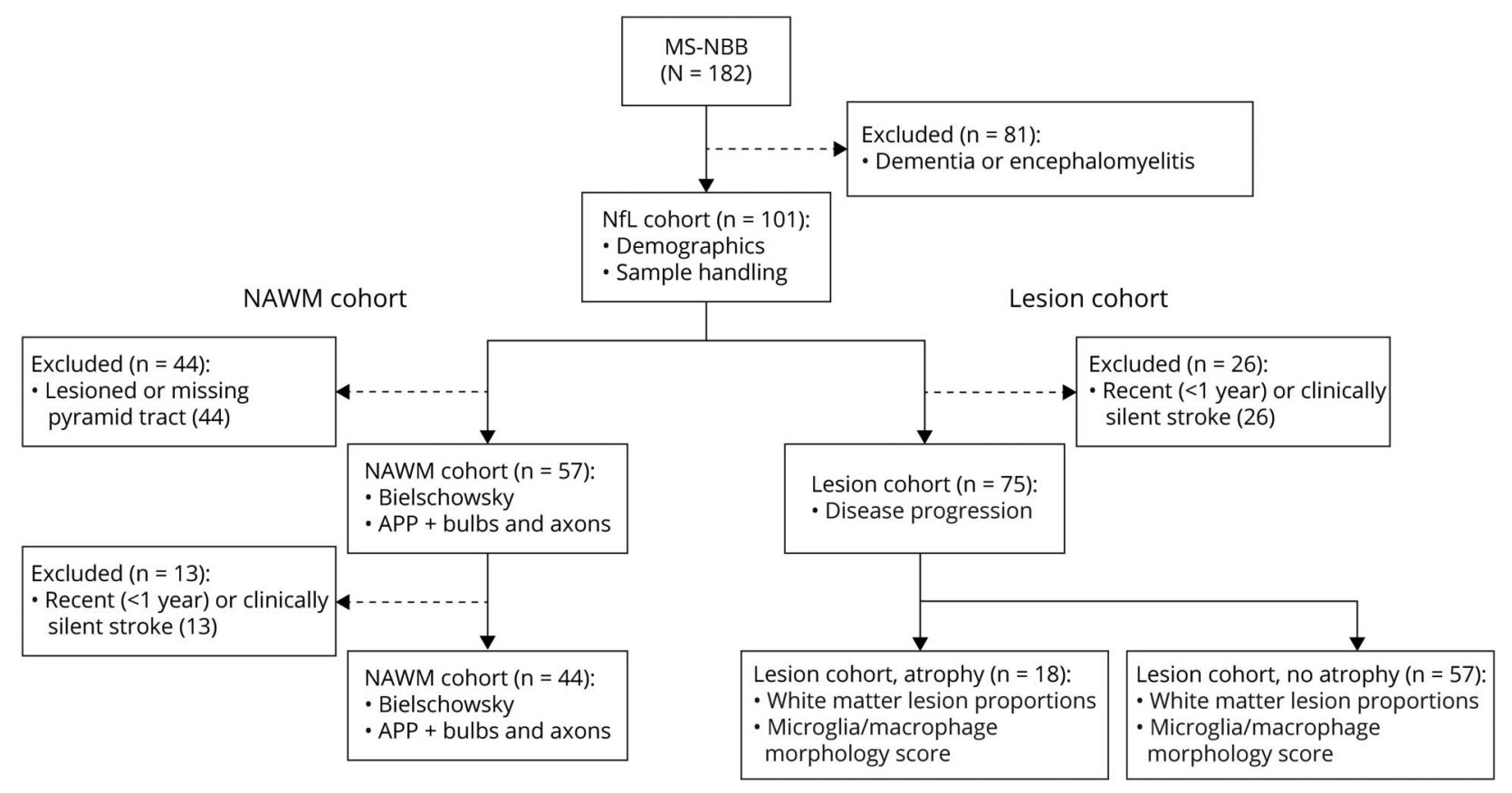

NAWM = normal appearing white matter.

macroscopically or on MRI guidance from $1 \mathrm{~cm}$ thick coronal brain slices cut throughout the brain. For the NAWM cohort (Figure 1), of all donors, the brain stem sample containing the pyramid tract was analyzed because this area is standardly dissected and therefore comparable between donors and because the axons are oriented in the same direction when cut longitudinal, facilitating comparable quantifications. Donors were excluded if there was a lesion present in the pyramid tract or if the pyramid tract was missing, resulting in an NAWM cohort comprising 57 donors.

\section{Neurofilament Measurements}

CSF $(n=101)$ and paired plasma samples $(n=43)$ were run simultaneously using a single molecule array (Simoa) assay to measure the NfL levels on an HD-X instrument (Quanterix, Billerica, MA) using the manufacturer's instructions. ${ }^{21,22}$ The Simoa assay is a highly sensitive sandwich-based enzyme-linked immune sorbent assay. The immunocomplex beads fit into 1 femtomolarsized chamber, resulting in highly concentrated reaction volume and signal per well. This technology allows an up to 1,000-fold increase in sensitivity, with multiple studies reporting strong correlations of CNS proteins between CSF and blood samples. ${ }^{24}$ Postmortem CSF and plasma samples were standardly diluted 1 : 100 and 1:4, respectively, and further diluted to conform the assay's dynamic range of $0.686-500 \mathrm{pg} / \mathrm{mL}$, with a dilution linearity (mean \%L between lower limit of quantification and upper limit of quantification) within the acceptable range of $85 \%-115 \%$.

\section{Lesion Characterization}

Characterization of MS lesions had been conducted previously for each donor on all available archived materials
$(3,819$ lesions in total) following the system previously described. ${ }^{3,26,27}$ Double immunohistochemistry for proteolipid protein (PLP) (MCA839G, AD Serotec, Oxford, UK, with $\mathrm{DAB}$ ) and human leukocyte antigen (HLA-DR-DQ) $(\text { M0775, CR3/43, DAKO, Denmark, with DAB + nickel })^{3}$ were performed. Reactive sites and white matter lesion types were discriminated based on demyelination and HLA-DR + microglia/macrophages. In reactive sites, there is no demyelination, and there is accumulation of microglia/ macrophages. In active lesions, there is partial demyelination and accumulation of microglia/macrophages throughout. In mixed lesions, there is a fully demyelinated, gliotic center with a border of accumulated microglia/ macrophages. The microglia/macrophages in active and mixed lesions are scored 0 if most of them are ramified, 0.5 if most of them are ameboid, or 1 if most of them are foamy. In inactive lesions, there is complete demyelination of the lesion, and there is no presence of microglia/macrophages. In remyelinated lesions, there is partial myelination, and there are sparse microglia/macrophages throughout. ${ }^{3,25,26}$

\section{Calculation of Lesion Load and Proportions of Lesions}

All white matter lesion parameters were calculated per donor previously. ${ }^{3}$ In the brainstem, a standardly dissected area, of each donor, the lesion load has been calculated as the sum of all white matter lesions present, and the reactive load has been calculated as the numbers of reactive sites identified. ${ }^{3}$ The MMAS of each donor has been calculated by dividing the sum of the scores of the microglia/macrophages values $(0,0.5$, and 1 ) in active and mixed lesions by the amount of active and 
Table 1 Multiple Sclerosis Donor Characteristics of the Various Subgroups Studied

\begin{tabular}{|c|c|c|c|c|c|c|}
\hline & \multicolumn{2}{|l|}{ All donors } & \multicolumn{4}{|c|}{ Donors without recent $(<1$ y) or clinically silent stroke } \\
\hline & All & $\begin{array}{l}\text { NAWM pyramid } \\
\text { tract present }\end{array}$ & All & Without atrophy & $\begin{array}{l}\text { With } \\
\text { atrophy }\end{array}$ & $\begin{array}{l}\text { NAWM pyramid } \\
\text { tract present }\end{array}$ \\
\hline & $N=101$ & $N=57$ & $N=75$ & $N=57$ & $N=18$ & $N=44$ \\
\hline Age $(y, S D)$ & $64.5(12.90)$ & $69.10(11.28)$ & $63.39(12.57)$ & $62.33(12.30)$ & $66.72(13.20)$ & $66.76(11.53)$ \\
\hline Sex (F\%) & 64.36 & 59.09 & 64.00 & 59.65 & 77.78 & 60.61 \\
\hline PMD (h, SD) & $8.01(2.70)$ & $7.81(3.18)$ & $7.80(2.72)$ & $7.91(2.83)$ & $7.46(2.41)$ & $7.83(3.43)$ \\
\hline $\begin{array}{l}\text { Brain weight } \\
(\mathrm{g}, \mathrm{SD})\end{array}$ & $1,182.4(135.86)$ & $1,198.53(136.48)$ & $1,168.89(134.53)$ & $1,202.09(122.88)^{\mathrm{a}}$ & $1,063.78(116.84)^{a}$ & $1,190.93(127.37)$ \\
\hline $\mathrm{pH}$ of CSF (SD) & $6.47(0.25)$ & $6.48(0.27)$ & $6.46(0.25)$ & $6.49(0.25)$ & $6.37(0.24)$ & $6.49(0.30)$ \\
\hline $\begin{array}{l}\text { Storage time } \\
(y, \text { SD) }\end{array}$ & $13.64(6.23)$ & $14.51(6.05)$ & $13.65(6.41)$ & $13.21(6.11)$ & $15.06(7.31)$ & $14.60(5.80)$ \\
\hline Age at onset (y) & $33.56(10.52)$ & 35.39 (11.57) & 33.54 (11.07) & 32.89 (10.79) & $35.5(11.98)$ & $35.03(11.85)$ \\
\hline Years to EDSS6 & $17.52(12.12)$ & 18.39 (11.57) & 16.85 (11.92) & $17.72(12.90)$ & $13.94(7.36)$ & $16.72(11.13)$ \\
\hline MMAS (SD) & $0.31(0.27)$ & $0.26(0.28)$ & $0.30(0.27)$ & $0.33(0.28)$ & $0.23(0.22)$ & $0.25(0.27)$ \\
\hline Lesion load BRS (SD) & $6.66(7.75)$ & $4.12(4.89)$ & $6.86(8.05)$ & $6.31(7.70)$ & $8.56(9.08)$ & $4.19(4.92)$ \\
\hline
\end{tabular}

Abbreviations: BRS = brain stem; EDSS6 = (Kurtze) Expanded Disability Status Scale; GLM = generalized linear model; MMAS = microglia/macrophage activation score; NAWM = normal appearing white matter; PMD = postmortem delay.

${ }^{a}$ Brain weight is significantly lower in donors with atrophy compared with that in donors without atrophy $(p=3.34 \mathrm{e}-5$, GLM).

mixed lesions. Proportions of active, mixed, and inactive lesions throughout the CNS were calculated per donor as the sum of the number of the specific type of lesions divided by the number of all lesions (active, mixed, inactive, and remyelinated lesions), discriminating between ramified, ameboid or foamy microglia/macrophages in active and mixed lesions. The proportion of remyelinated lesions was calculated per donor as the number of remyelinated lesions divided by the sum of inactive and remyelinated lesions. $^{3}$

\section{Quantification of Axonal Density and APP Presence}

To quantify axon density, Bielschowsky silver staining was performed on $8 \mu \mathrm{m}$ paraffin-embedded pyramid tract of the NAWM cohort $(n=57)$. Deparaffinization and rehydration was performed in a series of xylene and alcohol. Incubation with preheated $20 \%$ silver nitrate solution in $\mathrm{H}_{2} \mathrm{O}$ at $40^{\circ} \mathrm{C}$ was performed for 20 minutes. Tissue was placed in $\mathrm{H}_{2} \mathrm{O}$, and $32 \%$ ammonium hydroxide solution droplets were added before incubation with silver nitrate solution and incubated at $40^{\circ} \mathrm{C}$ for 20 minutes. Incubation with $1 \%$ ammonium solution was performed for 1 minute. Developer solution ( $50 \%$ nitric acid $(65 \%)+0.8 \%$ formaldehyde $(40 \%)$ $+0.2 \%$ citric acid in $\operatorname{depH}_{2} \mathrm{O}$ ) was added to the ammonium silver nitrate solution and incubated for 10 minutes. Five percentage sodium thiosulfate solution was incubated for 5 minutes, and dehydration was performed in alcohol series and xylene. Images were taken on an Axioskop (Zeiss) at 10x magnification, and automated random selection of ROIs comprising $>40 \%$ of the area was made, and the percentage of area covered by axons at $40 \times$ magnification was analyzed with the Image Pro Cell Count Grid software (Media Cubernetics, Rockville, MD).

For APP, ${ }^{27,28} 8 \mu \mathrm{m}$ paraffin-embedded NAWM subcortical white matter lesion $(n=50)$ sections were deparaffinized in a series of xylene and alcohol. Antigen retrieval was performed by microwaving citrate buffer $\mathrm{pH} 6.0$ for 10 minutes at $800 \mathrm{~W}$ before blocking with super mix (SUMI) $(0.25 \%$ gelatin + $0.5 \%$ Triton $\mathrm{X}+10 \%$ normal horse serum in tris buffered saline [TBS]). Primary antibodies (APP: MAB348 Millipore, $1: 1,000)$ were diluted in SUMI and incubated overnight. After blocking for endogenous peroxidase $\left(0.03 \% \mathrm{H}_{2} \mathrm{O}_{2}\right.$ in SUMI), biotinylated secondary antibody 1:400 in SUMI was incubated for 1 hour and avidin-biotin-complex (1:800 in TBS) was incubated for 45 minutes, visualized with $\mathrm{DAB}$ (50\% diaminobenzidine $+0.03 \% \mathrm{H}_{2} \mathrm{O}_{2}$ in TBS), and then counterstained with hematoxylin.

In the NAWM, donors were scored for the presence of APP + bulbs or axons. In the different white matter lesion types, the number of APP + bulbs and axon fragments relative to the area were counted, with the center and border of mixed lesions separately and in the perilesional white matter of the same tissue block. The counts were normalized to the APP + events in the perilesional white matter and to the area of the lesion. 
Table 2 Correlations of Clinical and Pathologic Donor Characteristics With CSF NfL Levels of All Multiple Sclerosis Donors

\begin{tabular}{|c|c|c|c|}
\hline & $\begin{array}{l}\text { CSF NfL mean (SD) } \\
\text { pg/mL (log) }\end{array}$ & $p$ Value & $\mathbf{r}$ \\
\hline Age & - & 0.85 & -0.02 \\
\hline \multicolumn{4}{|l|}{ Sex } \\
\hline Male & $7.57(1.25)$ & 0.35 & - \\
\hline Female & $7.75(1.23)$ & - & - \\
\hline pH of CSF & - & 0.25 & -0.12 \\
\hline Brain weight & - & 0.31 & 0.10 \\
\hline PMD & - & 0.38 & 0.09 \\
\hline Storage time & - & 0.36 & 0.09 \\
\hline Plasma NfL (pg/mL (log)) & - & $1.9 e-4$ & 0.54 \\
\hline \multicolumn{4}{|l|}{ Minor senile pathology } \\
\hline No & 7.71 (1.37) & 0.78 & - \\
\hline Yes & 7.66 (1.09) & - & - \\
\hline
\end{tabular}

Stroke (years from death)

\begin{tabular}{cllll}
\hline No & $7.25(0.98)$ & - & - \\
\hline Yes & & & \\
\hline$<\mathbf{1}$ & $9.42(1.40)$ & $\mathbf{2 . 4 e - 3}$ & - \\
\hline $\mathbf{1 - 5}$ & $7.47(0.87)$ & 0.46 & - \\
\hline$>\mathbf{5}$ & $7.68(1.33)$ & 0.72 & - \\
\hline Clinically silent & $8.50(1.13)$ & $3.5 e-4$ & \\
\hline
\end{tabular}

\section{Atrophy}

\begin{tabular}{llll}
\hline No & $7.73(1.27)$ & 0.31 & - \\
\hline Yes & $7.49(1.02)$ & - & - \\
\hline${\text { Age at onset }(\mathbf{y})^{\mathbf{a}}}$ & $33.53(11.07)$ & 0.33 & 0.12 \\
\hline Severity score $^{\mathbf{a}}$ & $3.85(0.31)$ & 0.16 & 0.16 \\
\hline${\text { Disease duration }(\mathbf{y})^{\mathbf{a}}}^{\text {A }}$ & $30.31(12.80)$ & $\mathbf{6 . 9 e - 3}$ & -0.31 \\
\hline
\end{tabular}

Abbreviations: $\mathrm{BRS}=$ brain stem; NfL = neurofilament light chain; $\mathrm{PMD}=$ postmortem delay.

Plasma NfL is positively correlated with CSF NfL. Compared with donors without stroke and those with stroke $>1$ y before death, recent $(<1 \mathrm{y})$ and clinically silent stroke increased NfL levels. In donors without recent and clinically silent stroke, CSF NfL levels negatively correlated with disease duration.

All associations were tested with the Pearson correlation coefficient. Wilcoxon rank sum test was used if 2 groups were compared and pairwise Wilcoxon rank sum test corrected for multiple testing for stroke at different time points.

CSF NfL levels were positively correlated to plasma NfL levels (1.9e-4), were significantly higher in donors with stroke $<1$ year prior to death $(p=2.4 \mathrm{e}-3)$ and clinically silent stroke (3.5e-4), and were negatively correlated to disease duration (6.9e-3).

${ }^{a}$ Donors without recent $(<1$ y) or clinically silent stroke.

\section{Statistical Analysis}

NfL measurements of the CSF and plasma, lesion, and reactive site load of the brainstem were natural log transformed to normalize the data for further analysis. Correlations between continuous variables were tested with the Pearson correlation coefficient. Differences between dichotomous variables and $\mathrm{NfL}$ concentrations were tested with Wilcoxon rank sum test. Differences between dichotomous variables and proportion measures were tested with binomial generalized linear models (GLMs). Correlations between proportion measures and continuous variables were tested with quasibinomial GLMs. Associations between dichotomous variables were tested with the Fisher exact test. If multiple groups were tested at once, a Kruskal-Wallis rank sum test was used in combination with pairwise Wilcoxon rank sum tests with corrections for multiple testing. All statistics were performed in RStudio Desktop, version 1.2.5033 (Rstudio, Inc, Boston, $\mathrm{MA})$, using key packages ggplot2, devtools, car, and lsmeans.

\section{Data Availability}

The data presented in this study are available on request from the corresponding author.

\section{Results}

\section{Donor Demographics and Sample Handling}

Correlations of CSF NfL levels with donor demographics and sample handling are summarized in Table 2. CSF NfL levels were not correlated with age, sex, $\mathrm{pH}$ of the CSF, weight of the brain, postmortem delay, or storage time. Compared with donors without stroke, NfL levels were significantly higher in donors with recent stroke $(<1$ year before death) (pairwise Wilcoxon rank sum tests with corrections for multiple testing, $p=2.4 \mathrm{e}-3)$ and with clinically silent stroke $(p=3.5 \mathrm{e}-4)$, and there were no differences found in donors with stroke $1-5$ years or $>5$ years before death. Because recent and clinically silent stroke elevate CSF NfL levels, this may confound the data. Therefore, for subsequent MS pathology-specific analysis, MS donors with recent $(<1$ year) and clinically silent stroke were excluded from further analyses (Figure 1, $\mathrm{n}=26$ excluded, $n=75$ remained). Minor senile pathology (Braak score 1-2 and/or Thal phase 1-2) did not correlate with NfL levels. Last, atrophy was validated by the lower brain weight of donors with atrophy than those without atrophy (Wilcoxon rank sum test, $p=7.4 \mathrm{e}-5$ ).

\section{Neurodegeneration}

\section{Clinical Disease Progression}

Correlations of CSF NfL with disease progression measures are summarized in Table 2. CSF NfL levels of MS donors at time of death did not correlate with age at onset of MS or severity score as calculated with time to EDSS 6, but did correlate negatively with the duration of disease, being higher in patients who exhibited a shorter disease duration (the Pearson correlation test, $p=6.9 \mathrm{e}-3, r$ $=-0.29)$, also after correction for age $(p=0.04, r=-0.24)$.

\section{Axonal Damage in the Normal Appearing White Matter}

Of 44 donors with MS, in the dissected medulla oblongate, the pyramid tract was missing or a lesion was present in the 
Figure 2 NfL Levels Are Negatively Correlated With Axonal Density and Are Increased With the Presence of Acute Axonal Damage in the NAWM
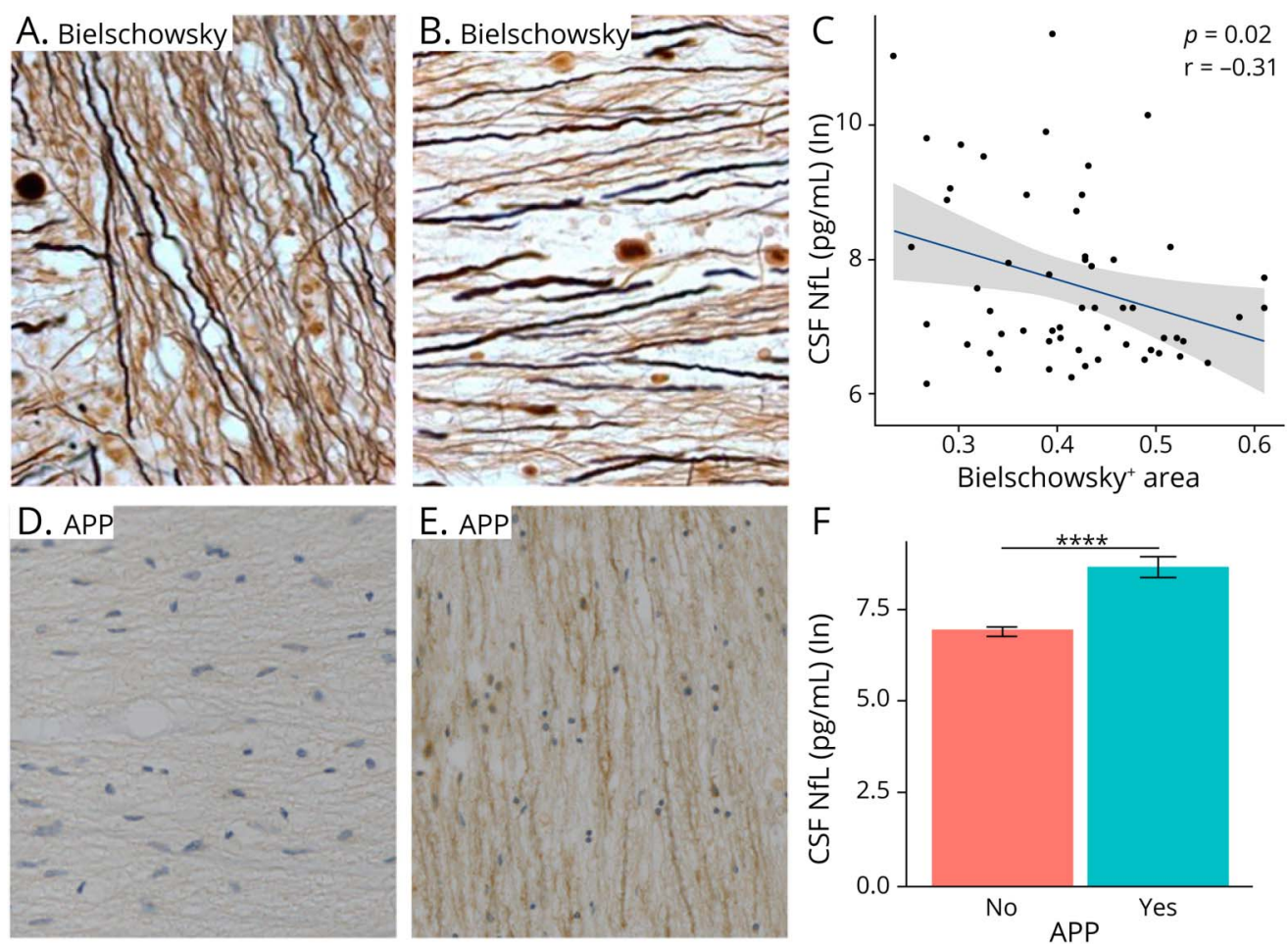

For axonal density, a Pearson correlation test was performed, and the regression line is visualized with the gray area indicating the $95 \% \mathrm{Cl}$. For APP+, a Wilcoxon rank sum test with correction of multiple testing was performed. Images are taken at 40x magnification. (A) Representative image of a high number of Bielschowsky + axons and (B) low number of Bielschowsky + axons. (C) Axonal density, as measured by Bielschowsky + axons, was negatively correlated with CSF NfL $(p=0.02, r=0.31)$. (D) Representative image of NAWM without APP + axons or bulbs, (E) Representative image of NAWM with APP + axons and bulbs. (F) The CSF NfL levels of donors with APP + axons or bulbs were significantly higher than the levels of those without APP + axons or bulbs ( $p=1.17 \mathrm{e}-6)$ $\mathrm{APP}=$ amyloid precursor protein; $\mathrm{NfL}=$ neurofilament light chain; NAWM = normal appearing white matter.

pyramid tract. In the remaining 57 donors, axonal density, quantified as the percentage of Bielschowsky positive area (high axonal density: Figure 2A, low axonal density: Figure $2 \mathrm{~B}$ ) in the NAWM, negatively correlated with NfL (Figure 2C, the Pearson correlation test, $p=0.02, r=-0.31$ ). In donors without recent or clinically silent stroke $(n=44)$, axonal density in the NAWM was not correlated with CSF NfL levels (data not shown).

In the NAWM $(n=57)$, the presence of APP + axons and bulbs was scored (no APP present: Figure 2D, APP + axons and bulbs present: Figure 2E). CSF NfL levels were significantly higher in donors with the presence of APP + axons or bulbs when compared with those without $(p=1.17 e-6)$. In donors without recent or clinically silent stroke $(n=44)$, donors with the presence of APP + axons or bulbs also had significantly higher CSF NfL levels when compared with donors without $\mathrm{APP}+$ axons or bulbs present (data not shown, $p=2.3 \mathrm{e}-4$ ).

\section{White Matter Inflammation and Lesion Activity} Because brain atrophy on MRI is related to NfL levels, ${ }^{9}$ we have analyzed correlations of CSF NfL in MS donors with macroscopic brain atrophy and without atrophy separately.
Donor demographics were not different between donors with atrophy and without atrophy, as summarized in Table 1. CSF NfL levels of MS donors without atrophy were similar to donors with atrophy (Wilcoxon rank sum test, Table 2), and donors with and without atrophy exhibited comparable lesion measures (Table 3).

Figure 3 shows ramified microglia/macrophages with MMAS score 0 (Figure 3A), foamy microglia/macrophages with MMAS score 1 (Figure 3B), microscopic images of an active lesion containing ramified (Figure 3C) and foamy (Figure 3D) microglia/macrophages, a mixed lesion containing ramified (Figure 3E) and foamy (Figure 3F) microglia/macrophages, an inactive lesion (Figure $3 \mathrm{G}$ ), and a remyelinated lesion (Figure $3 \mathrm{H}$ ). As listed in Table 3, correlations of CSF NfL with lesion measures found in all donors become stronger when donors with atrophy are removed. In donors without atrophy, CSF NfL levels correlated with several lesion type proportions.

In donors without atrophy, the MMAS positively correlated with CSF NfL (GLM, $p=1.2 \mathrm{e}-6$, Figure 3I). CSF NfL levels correlated with the proportion of all active lesions (GLM, 
Table 3 Correlations of Lesion Proportions and NfL Levels in the CSF in Multiple Sclerosis Donors Without Recent or Clinically Silent Stroke, Differentiated Between Donors With and Without Atrophy

\begin{tabular}{|c|c|c|c|c|c|c|c|c|}
\hline \multirow[b]{3}{*}{ Subtype } & \multirow{2}{*}{\multicolumn{3}{|c|}{ All $(n=75)$}} & \multicolumn{4}{|c|}{ Donors without recent or clinically silent stroke } & \multirow{3}{*}{$\begin{array}{l}\text { With vs } \\
\text { withou } \\
\text { atrophy } \\
p\end{array}$} \\
\hline & & & & \multicolumn{2}{|l|}{ With atrophy $(n=18)$} & \multicolumn{2}{|c|}{ Without atrophy $(n=57)$} & \\
\hline & $\begin{array}{l}\text { Total no. of } \\
\text { lesions }\end{array}$ & $\begin{array}{l}\text { Average load/ } \\
\text { proportion/score } \\
\text { (SD) }\end{array}$ & $p$ & $\begin{array}{l}\text { Average load/ } \\
\text { proportion/score } \\
\text { (SD) }\end{array}$ & $p$ & $\begin{array}{l}\text { Average load/ } \\
\text { proportion/score } \\
\text { (SD) }\end{array}$ & $p$ & \\
\hline Lesion load (BRS) & 659 & $1.51(1.14)$ & 0.36 & $1.65(1.27)$ & 0.37 & $1.46(1.10)$ & 0.68 & 0.55 \\
\hline $\begin{array}{l}\text { Reactive load } \\
\text { (BRS) }\end{array}$ & 149 & $0.55(0.74)$ & 0.85 & $0.73(0.87)$ & 0.20 & $0.49(0.68)$ & 0.47 & 0.24 \\
\hline Active & 874 & $0.21(0.23)$ & 0.01 & $0.18(0.22)$ & 0.64 & $0.22(0.23)$ & $6,35 \mathrm{e}-3$ & 0.51 \\
\hline Ramified & 299 & $0.08(0.15)$ & 0.33 & $0.09(0.16)$ & 0.73 & $0.08(0.15)$ & 0.31 & 0.70 \\
\hline Foamy & 356 & $0.07(0.13)$ & $6.25 e-10$ & $0.03(0.06)$ & 0.03 & $0.08(0.14)$ & $9.85 e-10$ & 0.14 \\
\hline Mixed & 985 & $0.27(0.27)$ & 0.50 & $0.22(0.29)$ & 0.15 & $0.28(0.27)$ & 0.14 & 0.40 \\
\hline Ramified & 416 & $0.13(0.17)$ & 0.43 & $0.07(0.08)$ & 0.41 & $0.14(0.19)$ & 0.41 & 0.08 \\
\hline Foamy & 194 & $0.04(0.06)$ & 0.02 & $0.02(0.05)$ & 0.13 & $0.04(0.07)$ & $1,75 e-3$ & 0.14 \\
\hline Inactive & 901 & $0.32(0.27)$ & 0.04 & $0.34(0.30)$ & 0.85 & $0.32(0.26)$ & $5,66 \mathrm{e}-3$ & 0.78 \\
\hline Remyelinated & 548 & $0.20(0.22)$ & 0.16 & $0.19(0.21)$ & 0.14 & $0.20(0.22)$ & 0.03 & 0.39 \\
\hline MMAS & 1,859 & $0.31(0.27)$ & 7.47e-7 & $0.23(0.22)$ & 0.28 & $0.33(0.28)$ & $1.2 \mathrm{e}-6$ & 0.07 \\
\hline
\end{tabular}

Abbreviations: BRS = brain stem; GLM = generalized linear model; MMAS = microglia/macrophage activation score; NfL = neurofilament light chain; PMD = postmortem delay.

Associations of lesion load and reactive load were tested with the Pearson correlation coefficient, and the rest were tested with quasibinomial GLMs. CSF NfL levels were positively correlated to the proportion of active lesions in all donors together $(p=0.01)$ and in donors without recent or clinically silent stroke without atrophy ( $p=6.35 \mathrm{e}-3)$. CSF NfL levels were positively correlated to the proportion of active lesions with foamy microlgia/macrophages in all donors together $(p=6.25 \mathrm{e}-10)$ and in donors without recent or clinically silent stroke with atrophy $(p=0.03)$ and without atrophy $(p=9.85 \mathrm{e}-10)$. CSF NfL levels were positively correlated to the proportion of mixed lesions in all donors together $(p=0.02)$ and in donors without recent or clinically silent stroke without atrophy $(p=1.75 \mathrm{e}-3)$. CSF NfL levels were negatively correlated to the proportion of inactive lesions in all donors together ( $p=0.04)$ and in donors without recent or clinically silent stroke without atrophy $(p=5.66 \mathrm{e}-3)$. CSF NfL levels were negatively correlated to the proportion of remyelinated lesions in donors without recent or clinically silent stroke without atrophy $(p=0.03)$. CSF NfL levels were positively correlated to the MMAS score in all donors together $(p=$ 7.47e-7) and in donors without recent or clinically silent stroke without atrophy $(p=1.2 \mathrm{e}-6)$.

$p=6.35 \mathrm{e}-3)$, and this correlation was considerably stronger for active lesions containing foamy microglia/macrophages (GLM, $p=9.85 \mathrm{e}-10$, Figure 3K). CSF NfL levels did not correlated with the proportion of all mixed lesions but was significantly correlated with the proportion of mixed lesions containing foamy microglia/macrophages (GLM, $p=1.75 \mathrm{e}-3$, Figure 3M). CSF NfL levels did not correlate significantly with the proportion of active or mixed lesions containing ramified microglia/macrophages (Figure 3, J and L). Reciprocally, CSF NfL levels negatively correlated with the proportion of inactive lesions (GLM, $p=1.75 \mathrm{e}-3$, Figure $3 \mathrm{~N}$ ) and remyelinated lesions (GLM, $p=0.03$, Figure 3O). Last, CSF NfL did not correlate with the lesion load or the proportion of reactive sites in the brain stem. In donors with atrophy, only a weak correlation was found of CSF NfL levels with the proportion of active lesions containing foamy microglia/macrophages (GLM, $p=0.03$ ), and no correlation was found with other lesion proportions.

\section{Plasma NfL Levels and White Matter Inflammation and Lesion Activity}

Regarding the more frequent use of plasma samples compared with CSF samples for NfL analyses in clinical monitoring of MS activity, we have explored the relation of plasma NfL with neuropathologic substrates in the small MS cohort with available plasma samples $(N=43)$. Unfortunately, the sample size of donors without recent $(<1$ year) or clinically silent stroke with plasma samples was low $(n=26)$. Plasma and CSF NfL levels correlated significantly (Figure 3P, the Pearson correlation test, $p=1.9 \mathrm{e}-4$, $r=0.54$ ). Correlations between plasma NfL levels and lesion load, reactive site load, lesion proportions, and the MMAS score are summarized in eTable 1 (links.lww.com/ NXI/A703). In donors without atrophy $(n=22)$, the correlation between plasma NfL and the MMAS score and the proportion of active lesions with foamy microglia/ macrophages showed generally similar trends as CSF NfL (eFigure 1).

\section{Acute Axonal Damage in Subcortical White Matter Lesions}

To confirm the association of foamy macrophages with increased axonal damage, as reflected by higher CSF NfL levels, we performed APP stainings in different lesion types. As visualized in Figure 4, in active lesions containing foamy microglia/macrophages, the amount of APP + bulbs and axon 

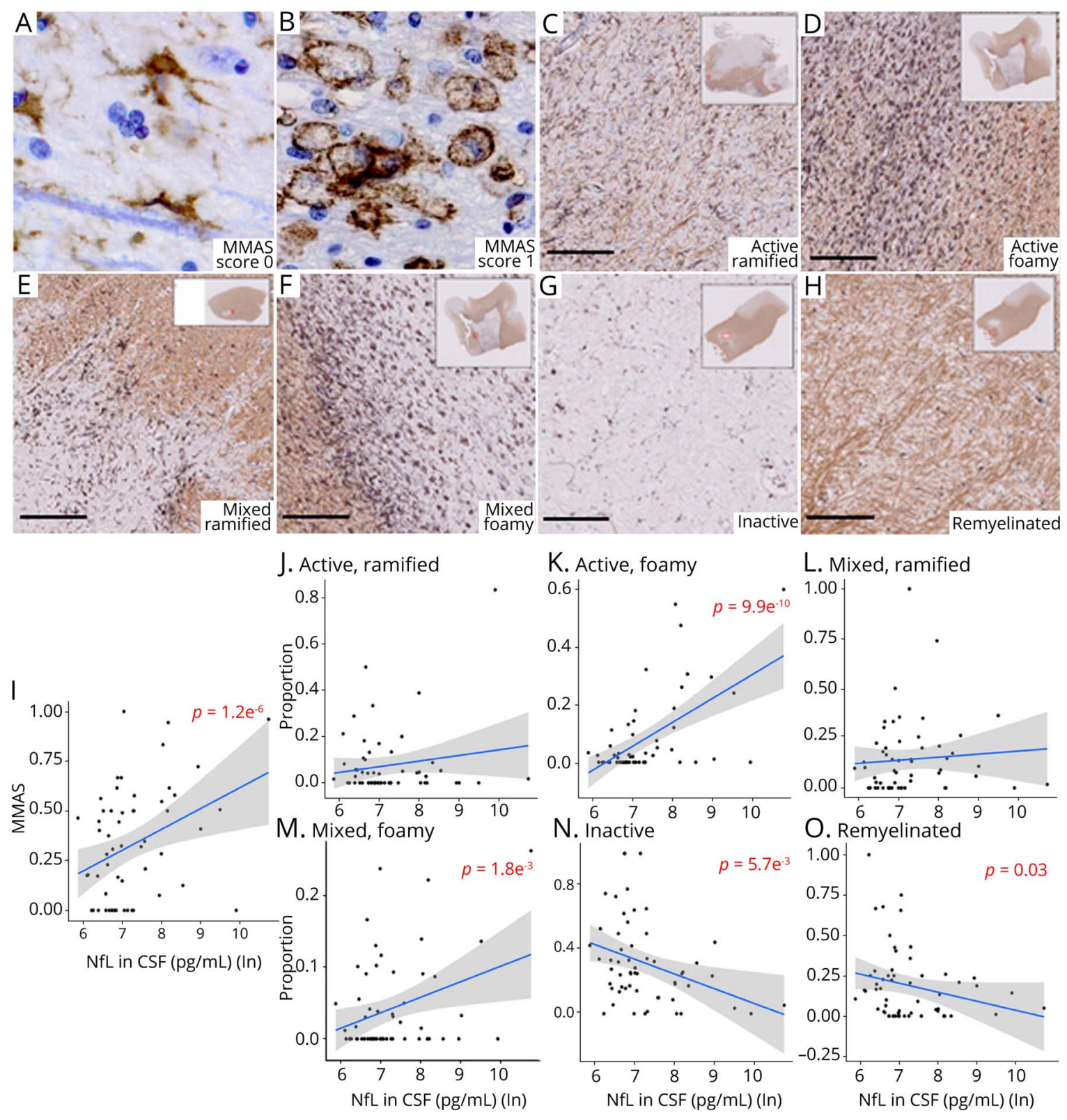

Correlations for different lesion measures were tested with a general linear model, and correlation between NfL levels in the CSF and plasma was tested with a Pearson correlation test, and the regression line is visualized with the gray area indicating $95 \% \mathrm{Cl}$. Representative immunohistochemical stainings show (A) ramified microglia/macrophages with MMAS 0 and (B) foamy microglia/macrophages with MMAS 1. Representative immunohistochemical stainings stained for PLP in brown and HLA-DR + microglia in black with a scale bar of $200 \mu \mathrm{m}$ of active lesions with (C) ramified microglia/macrophages, (D) foamy microglia/ macrophages, mixed lesions with (E) ramified microglia/macrophages and (F) foamy microglia/macrophages, (G) inactive lesions, and (H) remyelinated lesions. In donors without atrophy, CSF NfL levels positively correlated with (I) MMAS $(p=1.2 \mathrm{e}-6)$, and proportions of $(\mathrm{K})$ active lesions $(p=9.9 \mathrm{e}-10)$ with foamy microglia/macrophages, (M) mixed lesions with foamy microglia/macrophages $(p=1.8 \mathrm{e}-3)$, and CSF NfL levels negatively correlated with the proportions of $(\mathrm{N})$ inactive lesions $(p=5.7 \mathrm{e}-3)$ and $(\mathrm{O})$ remyelinated lesions $(p=0.03)$. HLA-DR = human leuykocyte antigen $-\mathrm{DR}$ isotype; MMAS = microglia/macrophage activation score; $\mathrm{NfL}=$ neurofilament light chain; PLP = proteolipid protein.

fragments, normalized to the amount of APP + bulbs and axon fragments in the perilesional white matter and the area of the lesion, was significantly higher compared with active lesions containing ramified microglia/macrophages (pairwise Wilcoxon rank sum tests with corrections for multiple testing, $p=$ 4.6e-3), active lesions containing ameboid microglia/ macrophages, the border and the center of mixed lesions containing ramified microglia/macrophages $(p=4.6 \mathrm{e}-3$ and $p=$ $4.6 \mathrm{e}-3$, respectively), the center of mixed lesions containing foamy microglia/macrophages $(p=4.6 \mathrm{e}-3)$, inactive lesions $(p=4.6 \mathrm{e}-3)$, and remyelinated lesions $(p=0.03)$. The amount of APP + bulbs and axon fragments was significantly higher in the border of mixed lesions containing foamy microglia/ macrophages compared with active lesions containing ramified microglia/macrophages $(p=0.02)$, the center of mixed lesions with ramified microglia/macrophages $(p=4.6 \mathrm{e}-3)$, the center of mixed lesions with foamy microglia $(p=0.02)$, inactive lesions $(p=4.6 \mathrm{e}-3)$, and remyelinated lesions $(p=0.04)$. 
Figure 4 Acute Axonal Damage Most Prevalent in Active Lesions With Foamy Microglia/Macrophages and the Border of Mixed Lesions With Foamy Microglia/Macrophages

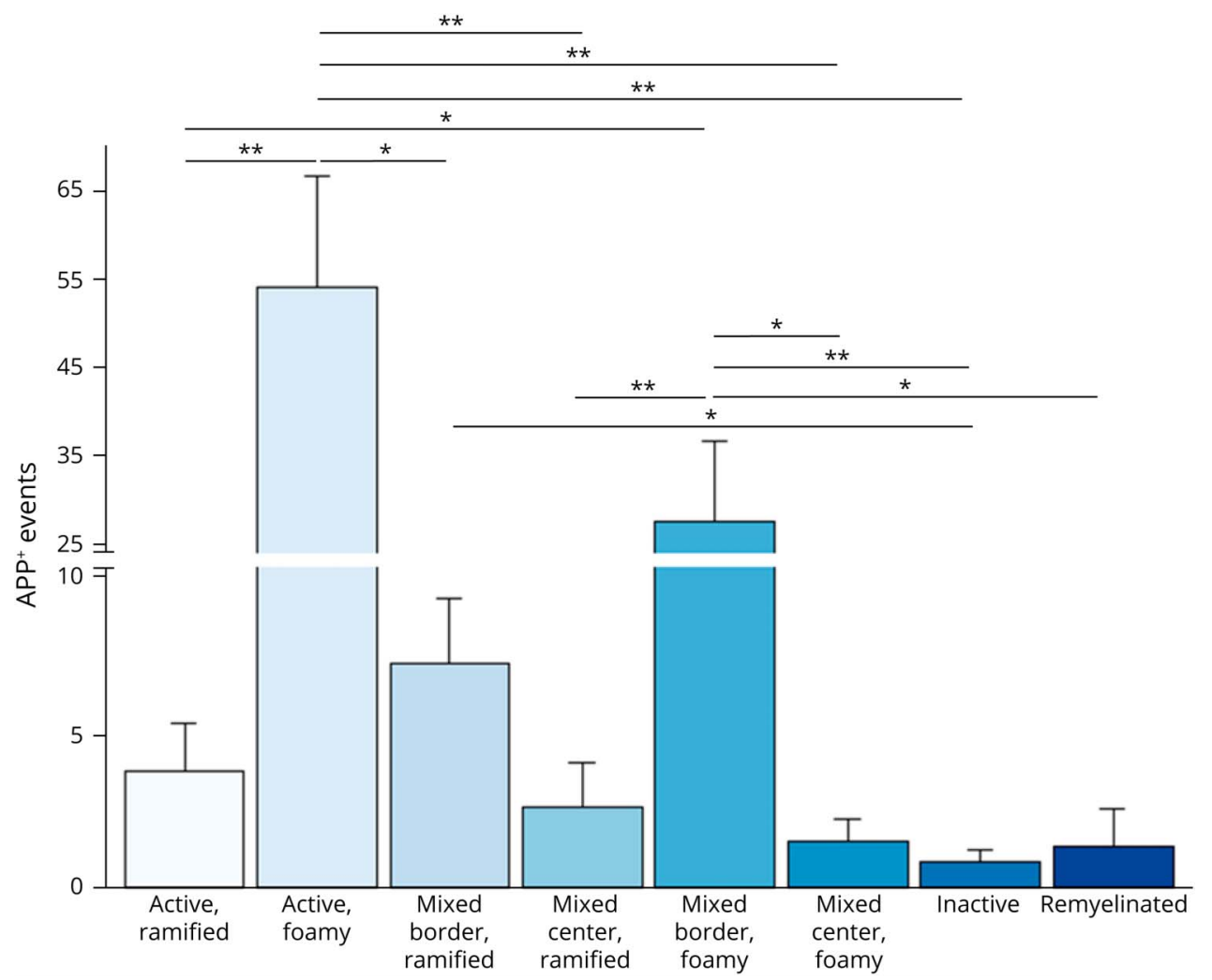

A Kruskal-Wallis test, followed by
pairwise Wilcoxon rank sum tests
with correction for multiple testing,
was performed. In active lesions
containing foamy microglia/macro-
phages, the amount of APP + bulbs
and axon fragments is significantly
higher compared with active lesions
containing ramified microglia/mac-
rophages $(p=4.6 \mathrm{e}-3)$, the border and
the center of mixed lesions contain-
ing ramified microglia/macrophages
$(p=4.6 \mathrm{e}-3$ and $p=4.6 \mathrm{e}-3$, respectively),
the center of mixed lesions containing
foamy microglia/macrophages $(p=$
$4.6 \mathrm{e}-3)$, inactive lesions $(p=4.6 \mathrm{e}-3)$, and
remyelinated lesions $(p=0.03)$. The
amount of APP + bulbs and axon
fragments is significantly higher in the
border of mixed lesions containing
foamy microglia/macrophages com-
pared with active lesions containing
ramified microglia/macrophages $(p=$
$0.02)$, the center of mixed lesions with
ramified and foamy microglia/macro-
phages $(p=4.6 \mathrm{e}-3$ and $p=0.02$, re-
spectively), inactive lesions $(p=4.6 \mathrm{e}-3)$,
and remyelinated lesions $(p=0.04)$.
The amount of APP + bulbs and axon
fragments in the border of mixed le-
sions containing ramified microglia/
macrophages is significantly higher
compared with inactive lesions $(p=$
$0.04)$. APP = amyloid precursor protein.

\section{Discussion}

In this study, we present a quantitative neuropathologic analysis of an extensive MS autopsy cohort of 101 brain donors and 3,819 lesions showing that acute axonal damage relating to active inflammatory lesion activity and reduced axonal density associate with increased CSF NfL levels. Our key findings are that (1) CSF $\mathrm{NfL}$ levels were negatively correlated with disease duration; (2) CSF NfL levels were positively correlated with increased axonal loss and with acute axonal damage in the NAWM; (3) CSF NfL levels were positively correlated with proportions of active and mixed lesions containing foamy microglia/macrophages and negatively correlated with inactive and remyelinating lesion proportions; and (4) active lesions with foamy microglia/ macrophages and the border of mixed lesions with foamy microglia/macrophages have a higher amount of APP + bulbs and axonal fragments when compared with active and mixed lesions containing ramified microglia/macrophages, inactive lesions, and remyelinated lesions. These findings show that specifically, MS lesions containing lipid-laden foamy microglia/ macrophages are associated with acute axonal damage and that proportion of such lesions positively correlate with CSF NfL levels. Furthermore, CSF NfL levels correlate with fast progression of MS. Together, this validates CSF NfL as a quantifiable biomarker for inflammatory white matter lesion activity-driven axonal damage and disease progression in MS.
In our cohort, CSF NfL levels were not confounded by donor demographics or by postmortem delay or storage time. In line with clinical studies, we found postmortem CSF NfL levels positively correlated with postmortem plasma NfL levels, with the same order of magnitude. ${ }^{19-21}$ In contrast to some previous studies, but in line with other clinical cohorts we did not observe an effect of age on CSF NfL levels in MS. ${ }^{12,14,18,19,21,29}$ Most likely, increases in CSF NfL due to acute axonal damage and demyelinating activity are superimposed on the increases due to ageing in our autopsy cohort. ${ }^{12}$ Similar to previous clinical studies, there were no sex-related differences in postmortem CSF NfL levels. ${ }^{14,15,19,21}$ The $\mathrm{pH}$ of the CSF, the brain weight, and the storage time did not correlate with CSF NfL levels, further validating our postmortem sampling method.

We confirmed a history of a recent stroke and a clinically silent stroke as confounding factors when studying MS-specific pathologies in relation to CSF NfL, and these donors were therefore excluded from MS-specific analyses. ${ }^{30-33}$ Brain atrophy in MS can also be a source of increases in NfL levels, ${ }^{23}$ but in our study, it did not have an effect on CSF NfL levels. Most likely, this is due to the spatial and temporal variations in atrophy that are not taken into account with the yes/no score used in this study. However, CSF NfL changes due to atrophy, the irreversible neurodegenerative component of $\mathrm{MS}^{34}$ superimposed on the effect of lesion characteristics on CSF 
NfL. Therefore, the relation between MS lesion characteristics and CSF NfL levels were analyzed separately in donors with and without atrophy in our study.

CSF and plasma NfL levels have previously been correlated with progression of MS-related disability during life. ${ }^{11,19,21}$ In this study, we showed that CSF NfL levels were negatively correlated with the disease duration reflecting a more severe course but not the age at onset or the disease severity score, as calculated with time to EDSS6. This suggests that donors with a shorter disease duration had experienced more neurodegeneration in the year before death compared with donors with a longer disease duration. CSF NfL levels reflect recent acute MS-related axonal damage, ${ }^{15,30}$ likely reflecting lesion activity causing axonal damage within a period of approximately the previous year, and are therefore not likely retrospectively reflecting the speed of disease progression before reaching EDSS6.

In the NAWM, CSF NfL negatively correlated with Bielschowsky + axons and therefore positively correlated with increased axonal loss. CSF NfL levels were significantly higher in donors with APP + axons or bulbs in the NAWM compared with donors without APP + axons or bulbs $(p=1.17 \mathrm{e}-6)$. This is most likely due to both Wallerian degeneration and neuroaxonal damage. 35,36

Previously, it was shown that APP + axons and bulbs are more frequently present in the border of mixed lesions than in the center. ${ }^{22,37}$ In this study, we corroborate these findings and additionally show in active lesions and the border of mixed lesions with foamy macrophages significantly more APP + acute axonal damage compared with active lesions and the border of mixed lesions with ramified microglia/macrophages. Therefore, the strong positive correlation between CSF NfL levels and the proportion of active and mixed lesions with foamy microglia/ macrophages is most likely due to the increased acute axonal damage in these lesions. The correlation of CSF NfL levels and proportions of (mixed) active MS lesions is in line with radiologic studies showing correlations between NfL with MRI biomarkers of inflammatory disease activity regarding the amount of gadolinium-enhancing lesions and paramagnetic rim MRI lesions. Of interest, our data suggest that foamy and ramified microglia may have different implications regarding neuroaxonal damage, likely relating to functional differences between lipidladen foamy vs ramified microglia/macrophages. ${ }^{38}$

Our study suggests several important issues about the use of CSF $\mathrm{NfL}$ as a biomarker for acute axonal damage in MS relating to lesion activity. First, we show that recent or clinically silent stroke and atrophy influence the relation between NfL CSF levels and MS lesion activity and acute axonal damage. Thus, these are comorbid conditions to use CSF NfL to monitor lesion activity and related axonal damage in patients with MS. Sequential NfL measurements within individual patients should solve this problem. Second, CSF samples were available of more brain donors than plasma samples, and therefore, we studied NfL levels in CSF. However, we showed that CSF NfL levels and plasma NfL correlate and that plasma NfL levels show a similar trend as CSF NfL levels toward a positive correlation with the MMAS score and the proportion of active lesions with foamy microglia/macrophages. Future studies should attempt to establish the relation of plasma NfL with neuropathologic substrates in MS because measurements of plasma NfL would be easier to apply clinically than those of CSF NfL. Last, due to a smaller group of donors with atrophy compared with donors without atrophy, it is possible that differences between these 2 groups are due to a loss of statistical power in the group with atrophy. However, because the relations between CSF NfL and lesion measures of all donors together are less significant than the relations between CSF NfL and lesion measures of only donors without atrophy, this suggests that these groups are fundamentally different.

In summary, in an MS autopsy cohort of 101 cases, we showed that CSF NfL levels negatively correlate with disease duration and positively correlate neuropathologically with proportions of active and mixed lesions containing foamy microglia/macrophages and acute axonal damage, validating $\mathrm{NfL}$ as a marker of disease activity.

\section{Acknowledgment}

The authors are grateful to the brain donors with multiple sclerosis who donated their brain for research and to the team of the Netherlands Brain Bank for their excellent service.

\section{Study Funding}

MS Research grants 17-975 and 19-1079 (MoveS).

\section{Disclosure}

The authors report no disclosures relevant to the manuscript. Go to Neurology.org/NN for full disclosures.

\section{Publication History}

Received by Neurology: Neuroimmunology \& Neuroinflammation October 25, 2021. Accepted in final form January 3, 2022.

Appendix Authors

\begin{tabular}{|c|c|c|}
\hline Name & Location & Contribution \\
\hline $\begin{array}{l}\text { Aletta van } \\
\text { den Bosch, } \\
\text { MSc }\end{array}$ & $\begin{array}{l}\text { Neuroimmunology } \\
\text { Research Group, } \\
\text { Netherlands Institute for } \\
\text { Neuroscience, Amsterdam, } \\
\text { The Netherlands }\end{array}$ & $\begin{array}{l}\text { Drafting/revision of the } \\
\text { manuscript for content, } \\
\text { including medical writing for } \\
\text { content; Major role in the } \\
\text { acquisition of data; Study } \\
\text { concept or design; Analysis } \\
\text { or interpretation of data }\end{array}$ \\
\hline $\begin{array}{l}\text { Nina Fransen, } \\
\text { PhD }\end{array}$ & $\begin{array}{l}\text { Neuroimmunology } \\
\text { Research Group, } \\
\text { Netherlands Institute for } \\
\text { Neuroscience, Amsterdam, } \\
\text { The Netherlands }\end{array}$ & $\begin{array}{l}\text { Major role in the acquisition } \\
\text { of data; Study concept or } \\
\text { design; Analysis or } \\
\text { interpretation of data }\end{array}$ \\
\hline $\begin{array}{l}\text { Matthew } \\
\text { Mason, PhD }\end{array}$ & $\begin{array}{l}\text { Neuroimmunology } \\
\text { Research Group, } \\
\text { Netherlands Institute for } \\
\text { Neuroscience, Amsterdam, } \\
\text { The Netherlands }\end{array}$ & $\begin{array}{l}\text { Study concept or design; } \\
\text { Analysis or interpretation of } \\
\text { data }\end{array}$ \\
\hline $\begin{array}{l}\text { Annemieke } \\
\text { Johanna } \\
\text { Rozemuller, } \\
\text { PhD }\end{array}$ & $\begin{array}{l}\text { Dept. Pathology, } \\
\text { Amsterdam UMC, } \\
\text { Amsterdam, The } \\
\text { Netherlands }\end{array}$ & $\begin{array}{l}\text { Major role in the acquisition } \\
\text { of data }\end{array}$ \\
\hline
\end{tabular}


Appendix (continued)

\begin{tabular}{|c|c|c|}
\hline Name & Location & Contribution \\
\hline $\begin{array}{l}\text { Charlotte } \\
\text { Teunissen, } \\
\text { PhD }\end{array}$ & $\begin{array}{l}\text { Neurochemistry lab, Dept. } \\
\text { Clinical Chemistry, } \\
\text { Amsterdam Neuroscience, } \\
\text { Amsterdam UMC, Vrije } \\
\text { Universiteit, Amsterdam, } \\
\text { The Netherlands }\end{array}$ & $\begin{array}{l}\text { Drafting/revision of the } \\
\text { manuscript for content, } \\
\text { including medical writing for } \\
\text { content; Major role in the } \\
\text { acquisition of data; Study } \\
\text { concept or design }\end{array}$ \\
\hline $\begin{array}{l}\text { Joost } \\
\text { Smolders, } \\
\text { PhD }\end{array}$ & $\begin{array}{l}\text { Neuroimmunology } \\
\text { Research Group, } \\
\text { Netherlands Institute for } \\
\text { Neuroscience, Amsterdam, } \\
\text { The Netherlands; Dept. of } \\
\text { Neurology and } \\
\text { Immunology, MS center } \\
\text { ErasMS, ErasmusMC, } \\
\text { Rotterdam, The } \\
\text { Netherlands }\end{array}$ & $\begin{array}{l}\text { Drafting/revision of the } \\
\text { manuscript for content, } \\
\text { including medical writing for } \\
\text { content; Study concept or } \\
\text { design; Analysis or } \\
\text { interpretation of data }\end{array}$ \\
\hline $\begin{array}{l}\text { Inge Huitinga, } \\
\text { PhD }\end{array}$ & $\begin{array}{l}\text { Neuroimmunology } \\
\text { Research Group, } \\
\text { Netherlands Institute for } \\
\text { Neuroscience, Amsterdam, } \\
\text { The Netherlands; } \\
\text { Swammerdam Institute for } \\
\text { Life Sciences, University of } \\
\text { Amsterdam, Amsterdam, } \\
\text { The Netherlands }\end{array}$ & $\begin{array}{l}\text { Drafting/revision of the } \\
\text { manuscript for content, } \\
\text { including medical writing for } \\
\text { content; Study concept or } \\
\text { design; Analysis or } \\
\text { interpretation of data }\end{array}$ \\
\hline
\end{tabular}

\section{References}

1. Lassmann H. Multiple sclerosis pathology. Cold Spring Harb Perspect Med. 2018;8(3):1-15.

2. Lassmann H, Raine CS, Antel J, Prineas JW. Immunopathology of multiple sclerosis: report on an international meeting held at the Institute of Neurology of the University of Vienna. J Neuroimmunol. 1998;86(2):213-217.

3. Luchetti S, Fransen NL, van Eden CG, Ramaglia V, Mason M, Huitinga I. Progressive multiple sclerosis patients show substantial lesion activity that correlates with clinical disease severity and sex: a retrospective autopsy cohort analysis. Acta Neuropathol .2018;135(4):511-528.

4. Stadelmann C, Wegner C, Brück W. Inflammation, demyelination, and degeneration recent insights from MS pathology. Biochim Biophys Acta. 2011;1812:275-282.

5. Frischer JM, Weigand SD, Guo Y, et al. Clinical and pathological insights into the dynamic nature of the white matter multiple sclerosis plaque. Ann Neurol. 2015;78(5):710-721.

6. Lucchinetti C, Brück W, Parisi J, Scheithauer B, Rodriguez M, Lassmann H. Heterogeneity of multiple sclerosis lesions: implications for the pathogenesis of demyelination. Ann Neurol. 2000;47(6):707-717.

7. Yuan A, Rao MV, Veeranna, Nixon RA. Neurofilaments at a glance. J Cell Sci. 2012; 125(14):3257-3263.

8. Ferreira-atuesta C, Reyes S, Giovanonni G, Gnanapavan S. The evolution of neurofilament light chain in multiple sclerosis. Front Neurosci. 2021;15(April):1-13.

9. Khalil M, Teunissen CE, Otto M, et al. Neurofilaments as biomarkers in neurological disorders. Nat Rev Neurol. 2018;14(10):577-589.

10. Ziemssen T, Akgün K, Brück W. Biomarkers in multiple sclerosis. J Neuroinflammation. 2019;9(3):1-11.

11. Varhaug KN, Torkildsen $\emptyset$, Myhr KM, Vedeler CA. Neurofilament light chain as a biomarker in multiple sclerosis. Front Neurol. 2019;10(April):1-6.

12. Uher T, Mccomb M, Galkin S, et al. Neurofilament levels are associated with blood brain barrier integrity, lymphocyte extravasation, and risk factors following the first demyelinating event in multiple sclerosis. Mult Scler. 2021;27(2):220-231.

13. Kapoor R, Smith KE, Allegretta M, et al. Serum neurofilament light as a biomarker in progressive multiple sclerosis. Neurology. 2020;95(10):436-444.
14. Bridel C, Van Wieringen WN, Zetterberg H, et al. Diagnostic value of cerebrospinal fluid neurofilament light protein in neurology: a systematic review and meta-analysis. JAMA Neurol. 2019;76(9):1035-1048.

15. Shahim P, Gren M, Liman V, et al. Serum neurofilament light protein predicts clinical outcome in traumatic brain injury. Sci Rep. 2016;6(November):36791-36799.

16. Zetterberg H, Skillbäck $\mathrm{T}$, Mattsson N, et al. Association of cerebrospinal fluid neurofilament light concentration with alzheimer disease progression. JAMA Neurol. 2016;73(1):60-67.

17. Gafson AR, Barthélemy NR, Bomont P, et al. Neurofilaments: neurobiological foundations for biomarker applications. Brain. 2020;143(7):1975-1998.

18. Kuhle J, Kropshofer H, Haering DA, et al. Blood neurofilament light chain as a biomarker of MS disease activity and treatment response. Neurology. 2019;92(10): E1007-E1015.

19. Piehl F, Kockum I, Khademi M, et al. Plasma neurofilament light chain levels in patients with MS switching from injectable therapies to fingolimod. Mult Scler. 2018; 24(8):1046-1054.

20. Barro C, Benkert P, Disanto G, et al. Serum neurofilament as a predictor of disease worsening and brain and spinal cord atrophy in multiple sclerosis. Brain. 2018;141(8): 2382-2391.

21. Disanto G, Barro C, Benkert P, et al. Serum Neurofilament light: a biomarker of neuronal damage in multiple sclerosis. Ann Neurol. 2017;81(6):857-870.

22. Maggi P, Kuhle J, Schädelin S, et al. Chronic white matter inflammation and serum neurofilament levels in multiple sclerosis. Neurology. 2021;97(6):e543-e553.

23. Kuhle J, Nourbakhsh B, Grant D, et al. Serum neurofilament is associated with progression of brain atrophy and disability in early MS. Neurology. 2017;88(9): 826-831.

24. Kuhle J, Barro C, Andreasson U, et al. Comparison of three analytical platforms for quantification of the neurofilament light chain in blood samples: ELISA, electrochemiluminescence immunoassay and Simoa. Clin Chem Lab Med. 2016;54(10): 1655-1661.

25. Kuhlmann T, Ludwin S, Prat A, Antel J, Brück W, Lassmann H. An updated histological classification system for multiple sclerosis lesions. Acta Neuropathol. 2017; 133(1):13-24.

26. Van Der Valk P, De Groot CJA. Staging of multiple sclerosis (MS) lesions: pathology of the time frame of MS. Neuropathol Appl Neurobiol. 2000;26(1):2-10.

27. Lindner M, Fokuhl J, Linsmeier F, Trebst C, Stangel M. Chronic toxic demyelination in the central nervous system leads to axonal damage despite remyelination. Neurosci Lett. 2009;453(2):120-125.

28. Nystad AE, Torkildsen $\varnothing$, Wergeland S. Effects of vitamin D on axonal damage during de- and remyelination in the cuprizone model. J Neuroimmunol. 2018;321(April): 61-65.

29. Bridel C, Verberk IMW, Heijst JJA, Killestein J, Teunissen CE. Variations in consecutive serum neurofilament light levels in healthy controls and multiple sclerosis patients. Mult Scler Relat Disord. 2020;47:102666.

30. Gattringer T, Pinter D, Enzinger C, et al. Serum neurofilament light is sensitive to active cerebral small vessel disease. Neurology. 2017;89(20):2108-2114.

31. Lewis SB, Wolper RA, Miralia L, Yang C, Shaw G. Detection of phosphorylated NF-H in the cerebrospinal fluid and blood of aneurysmal subarachnoid hemorrhage patients. J Cereb Blood Flow Metab. 2008;28(6):1261-1271.

32. Zanier ER, Refai D, Zipfel GJ, et al. Neurofilament light chain levels in ventricular cerebrospinal fluid following acute aneurysmal sybarachnoid hemorrhage. $2011 ; 8(2)$ : 157-159.

33. Uphaus T, Bittner S, Gröschel S, et al. NfL (neurofilament light chain) levels as a predictive marker for long-term outcome after ischemic stroke. Stroke. 2019;50(11): 3077-3084.

34. Andravizou A, Dardiotis E, Artemiadis A, et al. Brain atrophy in multiple sclerosis: mechanisms, clinical relevance and treatment options. Auto Immun Highlights. 2019; 10:7.

35. Allen IV, McQuaid S, Mirakhur M, Nevin G. Pathological abnormalities in the normal-appearing white matter in multiple sclerosis. Neurol Sci. 2001;22(2): 141-144.

36. Singh S, Dallenga T, Winkler A, et al. Relationship of acute axonal damage, Wallerian degeneration, and clinical disability in multiple sclerosis. J Neuroinflammation. 2017; 14(1):57-15.

37. Ferguson B, Matyszak MK, Esiri MM, Perry VH. Axonal damage in acute multiple sclerosis lesions. Brain. 1997;120 (pt 3):393-399.

38. Zia S, Rawji KS, Michaels NJ, et al. Microglia diversity in health and multiple sclerosis. Front Immunol. 2020;11(November):588021-588114. 


\title{
Neurology \\ Neuroimmunology \& Neuroinflammation
}

\author{
Neurofilament Light Chain Levels in Multiple Sclerosis Correlate With Lesions \\ Containing Foamy Macrophages and With Acute Axonal Damage \\ Aletta van den Bosch, Nina Fransen, Matthew Mason, et al. \\ Neurol Neuroimmunol Neuroinflamm 2022;9; \\ DOI 10.1212/NXI.0000000000001154
}

This information is current as of March 3, 2022

\section{Updated Information \& \\ Services}

References

Subspecialty Collections

Permissions \& Licensing

Reprints including high resolution figures, can be found at:

http://nn.neurology.org/content/9/3/e1154.full.html

This article cites 37 articles, 1 of which you can access for free at: http://nn.neurology.org/content/9/3/e1154.full.html\#\#ref-list-1

This article, along with others on similar topics, appears in the following collection(s):

Multiple sclerosis

http://nn.neurology.org//cgi/collection/multiple_sclerosis

Information about reproducing this article in parts (figures,tables) or in its entirety can be found online at:

http://nn.neurology.org/misc/about.xhtml\#permissions

Information about ordering reprints can be found online: http://nn.neurology.org/misc/addir.xhtml\#reprintsus

Neurol Neuroimmunol Neuroinflamm is an official journal of the American Academy of Neurology.

Published since April 2014, it is an open-access, online-only, continuous publication journal. Copyright

Copyright $\odot 2022$ The Author(s). Published by Wolters Kluwer Health, Inc. on behalf of the American

Academy of Neurology.. All rights reserved. Online ISSN: 2332-7812.

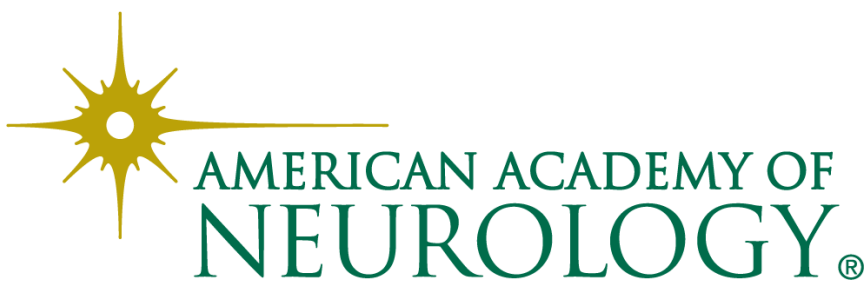

